\title{
Sisterhood? Jede für sich?
}

\author{
Gedanken über die heutige feministische Diskussion \\ in den USA
}

Die amerikanischen Feministinnen der 70er Jahre waren Idealistinnen. Sie waren politisch, aktivistisch, sie wollten die Gesellschaft ändern, und brauchten deshalb Parolen, sowie eine große gleichdenkende und -handelnde Gruppe, ein Wir, die unproblematisch ihre Identität ganz gezielt als Gruppe behaupten wollte. Ihre Parole "Wir sind alle Schwestern" erinnerte an Revolutionszeiten (als Analogie zur Brüderlichkeit der französischen Revolution); sie hat alle (oder zumindest viele) mitgerissen. Das andere Motto dieses feministischen Aufbruches, nämlich "das Private ist politisch «, war komplementär dazu, denn es deutete auf die Identität und die (fehlende) Repräsentation des weiblichen Geschlechts schlechthin: Wir Frauen, Bewohnerinnen der sogenannten persönlichen/privaten Sphäre, sind aber auch politisch, d.h. Teile der Öffentlichkeit, d.h. wir, als Gruppe verstanden, nehmen jetzt sehr klar an dem öffentlichen Leben teil.

Aber dieser zusammenfassende Begriff der "Sisterhood " war naiv und im Grunde höchst ethnozentrisch. So fragt Adrienne Rich, als sie zum wiederholten Mal eine Aussage über "wir Schwestern « oder »wir Feministinnen « hört: Who is We? (231). ${ }^{\prime}$ Die Idee eines undifferenzierten Ganzen, einer »Sisterhood «, taugt nicht, wenn wir statt an uns als Nur-Frauen mit einer gemeinsamen Identität zu denken, uns als Frauen mit sehr verschiedenen und manchmal widersprüchlichen Identitäten, Standpunkten, Eigenschaften, Herkünften betrachten. Begriffe wie Männlichkeit und Weiblichkeit, die zu diesem undifferenzierten Ganzen beigetragen haben, indem sie das Typische, das Allgemeine hervorgehoben haben, wurden eigentlich sehr früh von den Feministinnen als soziale Konstrukte und deshalb als unstabil und willkürlich erkannt. Und mit der zunehmenden Betonung der verschiedenen Identitäten von Frauen wurde die Politik des Ortes, des Standpunkts - identity politics - ins Zentrum des feministischen Projektes geriickt.

Adrienne Rich hat das schon 1984 eingesehen. In einer Rede, die sie damals vor einem europäischen Publikum gehalten hat, mußte sie, wohl mit Bedauern, das schöne Wort "Sisterhood « ersetzen, wenn sie versuchen wollte, den Stand des Feminismus um jene Zeit zu charakterisieren: Sie schlug also die "politics of location « als Begriff vor, ${ }^{2}$ einen Begriff, der heute nicht nur unter Feministinnen sondern auch in der sogenannten »multiculturalism «-Debatte in Amerika eine große Bedeutung hat. Im folgenden möchte ich die wachsende Spannung zwischen 
dieser "politics of location « - die sehr nahe an »identity politics" grenzt - und der wachsenden Reaktion gegen den vor allem von postmodernen Feministinnen behaupteten Anti-Essentialismus darstellen, besonders in bezug auf die Infragestellung dieser zwei Begriffe.

\section{Beispiele derzeitiger Frage- und Problemstellungen im amerikanischen Feminismus}

Aus den zur Zeit wuchernden komplexen Debatten im amerikanischen Feminismus möchte ich fünf Fragestellungen herauskristallisieren, die teilweise auf die gleichen Problemlagen reagieren.

1. die Frage von inside und outside:

Früher waren die Verhältnisse einfach: Die Feministinnen waren outside, die Männer (oder die traditionellen DenkerInnen) waren inside. ${ }^{3}$ Aber heute, nachdem die akademische Institutionalisierung des Feminismus in den USA stattgefunden hat, gibt es eigentlich eine neue Auffassung des inside und outside: Inside sind die privilegierten weißen akademischen feministischen Wissenschaftlerinnen, outside sind immer noch die meisten »women of color «, die marxistischen und lesbischen Feministinnen (die auch z.T., sogar wenn sie inside, d.h. in der Institution sind, sich jedoch outside befinden, d.h. in der Forschungspraxis auch inhaltlich marginalisiert werden). Diese neue Spannung wirkt sich auf die Theoriebildung aus: Diejenigen, die meist noch outside sind (oder innerhalb der Hochschulen in einer outside-Position stehen), bezeichnen sich als Aktivistinnen, wenn nicht als AntiTheoretikerinnen, wenigstens versuchen sie, eine andere Art von eher praxisbezogener Theorie zu entwickeln.

Dieses räumliche Schema (inside-outside) provoziert die Frage nach der Marginalität, wobei sie jedoch aufgewertet statt entwertet und immer weniger mit Frauen als solchen und immer mehr mit "people of color « (also nicht ausschlieBlich Frauen) und anderen heutzutage als outside Gekennzeichneten identifizien wird. Eine bewußte, positive, aktive Besetzung dieses Ortes wird vorgenommen, es wird von einer »decentering of the center " gesprochen, und der Outsider-Status wird dabei nicht nur umgewertet, sondern in mancher Hinsicht sogar privilegiert. Und theoretisiert: Die Philosophin Diana Fuss versucht, eine Theorie der Marginalität zu entwickeln, was sie "a theory of marginality, subversions, dissidence, and othering ... a theory of sexual borders « nennt. ${ }^{4}$ (5) Und andere, zum Beispiel bell hooks, schreiben über marginale Orte als "spaces of radical openness «: »Without such spaces we [d.h. people of color] would not survive. Our living depends on our ability to conceptualize alternatives, often improvised. Theorizing about this experience aesthetically, critically is an agenda for radical cultural practice. $\aleph^{5}$ (»Choosing the Margin «, 148-49).

2. Eine große Diskussion, auch unter denen, die inside und/oder outside sind, befaßt sich immer noch mit der Differenz. Früher wurde die Differenz innerhalb einer rein geschlechtlichen/biologischen Terminologie interpretiert, nämlich zwi- 
schen Männern und Frauen. Im Zuge der Entwicklung der Postmoderne wurde diese Diskussion modifiziert, als die Behauptung, alles sei sozial konstruiert, verallgemeinernde Kategorien wie z.B. Männlichkeit und Weiblichkeit immer mehr infragegestellt hat. In der Folge wurde das Wort "Differenz « in Amerika vor allem von »women of color « immer öfter bezogen ausschließlich auf die Differenzen unter Frauen. Daraus entstand eine breit angelegte Diskussion um identity politics, ein Teil der schon erwähnten Entwicklung der neuen Insider und Outsider Gruppen. Innerhalb der Diskussion jedoch gibt es deutliche Unterschiede: Theoretisch wird "Differenz « an der Universität von den insiders diskutiert, erlebt und erzählt dagegen wird die Differenz von seiten der »anderen Frauen « außerhalb der Universität (oder marginalisiert innerhalb der Universität). Wiederum also geht es wenigstens zum Teil um eine verschobene Theorie - vs. Praxis-Diskussion.

Zusätzlich wird die Politik der Differenz diskutiert, wie etwa von der schwarzen Anglistin Hazel Carby, die dem Feminismus vorwirft, die Diskussion der Differenz viel zu eng zu gestalten. Dabei sei an Dominanzstruktur zu denken, nicht Vielfalt (diversity): Denn in den Vorstellungen über diversity sei die Kategorie der Rasse marginalisiert und werde nur dann miteinbezogen, wenn über schwarze (oder andere farbige) Menschen gesprochen werde - als ob weiß keine Farbe sei. $\mathrm{Da} B$ dabei die Machtverhältnisse meist ignoriert werden, zeigt Carby außerdem an einem anderen Beispiel: Wir integrieren schwarze Frauen in den Lehrplan, aber wir ignorieren meist völlig die materiellen Bedingungen der meisten Schwarzen. Carby empfiehlt also, da $B$ wir die Existenz von historisch spezifischen Formen des Rassismus anerkennen; da $\beta$ wir an Rassismen statt an nur einen Rassismus denken; $\mathrm{da} B$ wir verschiedene ethnische Gruppen miteinbeziehen statt uns nur zeitlose essentialistische Rassen vorzustellen. (10) Und sie betont vor allem die Politik: Sie bemerkt z. B., daß Theorien von Differenz oder von diversity uns in der Praxis fragmentiert und getrennt lassen, "gleich « seien wir nur in unserem Unvermögen, uns radikale gesellschaftliche Änderungen vorzustellen.

3. Auch damit verbunden ist eine Kritik der Aussagen der postmodernen Feministinnen, die eine Art vereinfachte Essentialisierung des Essentialismus anbieten: Ihnen wird vorgeworfen, keine Alternativen zum Essentialismus, d.h. zu der Idee eines weiblichen/biologischen "Wesens « anzubieten, sondern eher ein Bild des Nichts. Es wird gefragt, wenn es keine weibliche »Essenz « gibt, was es denn sonst sei, was uns zu Frauen macht? Ist "Frau« nichts als ein soziales Konstrukt? Einwände gegen die postmodernen Feministinnen werden zum Beispiel von Teresa de Lauretis und Marilyn Frye formuliert (vgl. unten).

4. Die Diskussionen um die immer noch als problematisch betrachtete Institutionalisierung des Feminismus in den USA dauern an. Erneut geht es um die Frage Theorie vs. Praxis bzw. um eine Auseinandersetzung über die verschiedenen Arten von Theorie, die nicht ausschließlich in der akademischen Welt entwickelt werden oder von den üblichen akademischen Theorien abweichen. Die Frage um das Verhältnis von Theorie und Praxis geht immer noch darum, ob sie komplementär sind oder einander ausschließen bzw. wie wir sie innerhalb der Universität am besten miteinander kombinieren können, um die Dichotomie aufzulösen. 
5. Ein Konflikt, der der Diskussion über Differenz komplementär ist, leitet eine Differenzierung in diese Diskussion ein: nämlich der Konflikt zwischen dem Drang nach »Multiculturalism* (d.h. dem Drang nach einer Partikularisierung einzelner Kulturen statt der früher üblichen Idee des »melting pot«) und den oft ziemlich starren gender-spezifischen Erwartungen der einzelnen Kulturen gegenüber ihrer eigenen Selbstdefinition. Grob gesagt, die Achtung vor einzelnen Kulturen steht im Widerspruch zu den oft frauenfeindlichen Werten in diesen Kulturen. Ein Beispiel ist die Diskussion über Klitorisbeschneidung. Noch gröber gesagt, ein Konflikt zwischen Kultur und Frauen, wobei die Kultur als männlich genormt verstanden wird und die Frauen innerhalb dieser Kultur in einer rigiden Rollenerwartung gefangen sind.

Was allen diesen Fragenkomplexen gemeinsam ist, ist die Frage des "Ortes«, der location, bzw. der Identität. Es geht hier um den Platz ( $\mathrm{Ob}$ inside oder outside, ob "woman of color «, ob Lesbe, ob weiße Frau, ob Frau aus dem Arbeiterstand etc.) - noch mehr, um einen Aspekt unserer persönlichen und politischen Selbstbehauptung, da wir unsere Identität mit unserem Standpunkt verbinden. ${ }^{6}$ Hier geht es auch beinahe ausschließlich um Frauen: Diese fünf Fragestellungen befassen sich vor allem mit dem Geschlecht als ausschlaggebender analytischer Kategorie.

\section{II. (Anti-)Essentialismus und Identitätspolitik und die Art ihrer Infragestellung}

Auf zwei dieser Fragenkomplexe möchte ich jetzt näher eingehen, nämlich die Diskussionen um den Anti-Essentialismus (Punkt 3) und die Debatten um die identity politics (die in allen erwähnten Fragestellungen eine Rolle spielen). Es geht in beiden Fällen um die Politik des Ortes: Wo stehen wir, woher kommen wir, und welche Rolle spielt das in der Entwicklung der feministischen Theorien? Aber auch: Gibt es überhaupt ein Wir (hier verstanden als politische Einheit)? oder heutzutage nur ein Ich? (oder, wenn wir der Postmoderne zuhören, nicht einmal das?)

Der Begriff Essentialismus wird vor allem auf die ziemlich begrenzte biologische Ebene bezogen, d.h. auf »sex «, aber nicht ausschließlich darauf. In bezug auf den Feminismus heißt es auf der einen Seite, daß alle Frauen gleich sind, daß es tatsächlich eine weibliche Identität gibt, biologisch (sex) sowie gesellschaftlich (gender), die allen Frauen gemeinsam ist: eine Essenz, die wir als Frauen besitzen. Es gibt bestimmt essentialistische Echos in dem frühen Ausruf der Feministinnen, »sisterhood is powerful«, nämlich da $\beta$ wir alle Schwestern (d.h. gleich) sind, da $\beta$ wir alle sowohl biologische als auch gesellschaftliche Aspekte gemeinsam haben. In anderen Worten, daß das Geschlecht die Grundlage unserer Existenz bildet. Auf etwas gröbere Weise gesagt, stammt die (moderne) Idee des gendered Essentialismus aus den Begriffen Männlichkeit und Weiblichkeit, die schon im späten 18. Jahrhundert als feste Kategorien verstanden wurden, mit zeitlosen (absoluten) Komponenten ("Geschlechtscharaktere «), mit festen Essenzen und Aspekten, die über Zeiten und Kulturen fortdauern sollten. Aber sogar wenn wir von der Biologie 
und vom Gender absehen, gibt es andere Arten von Essentialismus, nämlich diejenigen unter verschiedenen Gruppen, Lesben, Afro-AmerikanerInnen etc., die aus anderen Kategorien wie sexueller Orientierung oder Rasse herstammen, die ebenfalls behandelt werden, als ob sie feste Essenzen in sich enthalten; sie werden immer im Prozeß des Selbstdefinierens benutzt.

Identity politics stellt den Essentialismus infrage, insofern in ihn "Identität «, Differenz und Gegensätzlichkeit impliziert sind: Wir sind nicht nur als Frauen und Männer anders, sondern auch unter Frauen (bzw. unter Lesben, unter Afro-Amerikanerinnen etc.); es gibt nicht nur den Faktor Gender, sondern auch Rasse, Klasse, sexuelle Orientierung etc. und deren verschiedenen Formen, Kategorien, die uns eher voneinander trennen als miteinander verbinden.

Ich werde hier an die Herausforderung an den weißen bürgerlichen Feminismus erinnert (in den USA stammt sie vor allem von »women of color «), die eigene Identität ebenso wie die von anderen Frauen zu problematisieren. Der Begriff des Feminismus wird immer noch als weiB und bürgerlich verstanden: Es waren die Feministinnen in den 70er Jahren, die nicht nur tatsächlich weiß und bürgerlich waren, sondern auch immer wieder von der globalen oder wenigstens der allgemeinen "Sisterhood « gesprochen haben; heutzutage wird sogar das Wort »Feministinnen « von manchen »women of color « als implizite Bezeichnung für eine ausschließlich weiße Gruppe von Frauen gebraucht. Die Differenz wird nicht mehr als ausschließlich gender-spezifisch verstanden, sondern als viel komplizierter, auch viel widersprüchlicher und schwieriger. Die Idee der »multiple identities«, oft in einem einzigen Menschen im Widerspruch miteinander, wird wichtig. Dabei wird auch von manchen das Individuum betont, das Partikuläre an einem Menschen, ob Frau, ob Mann. Hervorgehoben wird auch ein Begriff wie »situated knowledges " (und auch "partially situated knowledges «; vgl. Haraway). Der Ort ist auf jeden Fall entscheidend, das Räumliche (mehr wohl als das Zeitliche) wird betont. Welche verschiedenen kontextuellen Faktoren haben uns als Frauen geprägt, aus welcher spezifischen Perspektive betrachten wir unsere Umgebung? Und welcher unter diesen Faktoren wird bevorzugt?

Zwei Beispiele der identity politics bieten Aufsätze von Minnie Bruce Pratt und Gloria Anzaldúa. Pratts Aufsatz, "Identity: Skin Blood Heart «, beschreibt ihren schwierigen Versuch, ihre Identität zu suchen und zu begreifen, wobei sie anerkennen mu $B, \mathrm{da} B$ es ein unendlicher Proze $B$ ist, der immer weiter differenziert werden muß. Es genügt ihr nicht, sich als Frau zu identifizieren, es kommen viele andere Schichten dazu, negativ sowie positiv: Sie erkennt sich also langsam und mit großer Mühe an als weiße, bürgerliche, protestantische, aus den Südstaaten stammende Frau, als Ehefrau, als Mutter, aber auch als Lesbe, auch als rassistisch, als feministisch, als politisch engagiert usw.

Für Gloria Anzaldúa, die in ihrem Aufsatz ihre Identität als lesbische Schriftstellerin und "woman of color « zu erklären versucht, ist die Bezeichnung einer location ausschlaggebend: Das Wort lesbos z. B. sei griechisch, europäisch, sagt sie, habe also nichts mit ihr als Chicana (eine mexikanisch-amerikanische Frau) zu tun, sei eine Art Kolonisierungswort: "yes, we may all love members of the same sex but we are not the same. $\ll^{7}(250)$ Aber Anzaldúas Grund, sich ausführlich und spezifisch zu identifizieren, hat wenig mit dem Ziel der dominanten Kultur 
zu tun, die sie von außen her kennzeichnet, um sie zu marginalisieren oder zu begrenzen: »My labeling of myself is so that the Chicana and the lesbian and all the other persons in me don't get erased, omitted or killed. Naming is how I make my presence known, how I assert who and what I am and want to be known as. Naming myself is a survival tactic. $\otimes^{8}(251)$ Für sie ist das Sich-Identifizieren eher ein fließender Proze $B$ als eine Konkretisierung: Das heißt, in gewisser Hinsicht löst sie das Problem der identity politics auf, indem sie den Prozeß der Identifizierung anders formuliert, indem sie ihn als Ineinanderfließen, als Grenzenüberspringen betrachtet, nicht etwa als einen Isolierungsproze $B$. Bei ihr also wäre die Politik immer noch möglich, die Koalitionen auch, nur anders formuliert und verstanden.

In beiden Fällen wird das Ich untersucht, zum Teil definiert, aber eigentlich vor allem komplexer, problematischer gemacht: Die Identitätssuche bringt also einen Prozeß der wachsenden Differenzierung mit sich, schließlich resultiert daraus eher eine Multiplizität des Ichs statt eines vereinten Selbstbildes. Es kann beruhigend wirken: Die persönliche, die subjektive Stimme innerhalb eines Textes (ob kritisch oder literarisch) war ja immer ein Grundelement der feministischen Diskussion. Wir haben schon lange dafür plädiert, daß wir unser Ich ausdrücken wollen, wir haben die sogenannte Objektivität des akademischen Diskurses immer wieder infragegestellt. Aber hier ist es noch verwickelter: Ein Ich auszudrücken heißt noch lange nicht, daß wir dieses Ich voll und ganz verstehen oder umschreiben können. Insofern müssen wir uns fragen, wo wir bei einer immer stärker differenzierten »location « bzw. »Identität« landen: Ist es der Schnittpunkt aller unserer Gruppenzugehörigkeiten? Und wie sind diese im Verhältnis zueinander gewichtet, was ist »authentisch«, und gibt es überhaupt eine »authentische« Identität?

\section{Weitere Problematisierungen dieser Begriffe}

Während Vertreterinnen der Postmoderne (und darunter sind auch viele Feministinnen, z. B. Linda Nicholson, Nancy Fraser, Chris Weedon) »Essentialismus« im negativen Sinne gebrauchen, gibt es auch andere (z.B. Teresa de Lauretis, Vicki Kirby, Elizabeth Grosz, Marilyn Frye), die nicht bereit sind, den Begriff der Essenz ganz aufzugeben, d.h., die sich verweigern, essentialistisch als undifferenziertes Verdammungswort anzunehmen, während es keine politisch zufriedenstellenden Alternativen gibt. Sie betrachten ja den Essentialismus, wenigstens zum Teil, als mögliche politische Strategie. Manche, wie Teresa de Lauretis und Elizabeth Grosz, wollen das Wort selber präziser gebrauchen, indem sie es von verwandten (Schimpf-)Wörtern wie Naturalismus, Universalismus und Biologismus unterscheiden; manche andere, wie Marilyn Frye, finden den Essentialismusvorwurf auf seiten der postmodernen Feministinnen verdächtig, »not politically innocent « (4), indem Ideen pauschal als essentialistisch verdammt werden, ohne daß klar werde, was daran »essentialistisch« und warum das schlecht sei. Noch gezielter schreibt Tania Modleski in ihrem neuen Buch mit dem provokativen Titel Feminism Without Women: Culture and Criticism in a "Postfeminist Age: 
I worry that the position of female anti-essentialism as it is being theorized by some feminists today is a luxury open only to the most privileged women. I worry about the consonance of this position with the ones being advanced by certain white male poststructuralist intellectuals who have proclaimed the death of the subject: if Nancy $K$. Miller is correct to counter proclamations about the death of the subject by insisting that »only those who have it can play with not having it, "could we not also say of anti-essentialist feminists that only those possessing vastly wider options than the majority of women living in the world today can play at "being it" without theorizing themselves into the belief that they are not it? $(22)^{9}$

Noch andere Wissenschaftlerinnen befassen sich mit einer etwas skeptischen Auffassung der anti-essentialistischen Aussagen. ${ }^{10}$ Wie Modleski behauptet auch Vicki Kirby u.a., daß die Idee von gut (Anti-Essentialismus) und böse (Essentialismus) aufrechtzuerhalten »a dubious moral agenda " anbietet. (7) Sie interpretiert das Negieren vom Essentialismus sogar als Negierung vom Feminismus: Die Dichotomisierung zwischen Essentialismus und Anti-Essentialismus, sagt sie, "also denies feminism's achievements and the mutability of feminism's own ident- ity in negotiating and challenging the different meanings that inform this division within shifting historical contexts. « ${ }^{11}$ (7) Elizabeth Grosz differenziert zwischen einer Reihe von verwandten Begriffen, während Marilyn Frye den Essentialismusvorwurf als auf einem meist falschen Gebrauch des Wortes beruhend bezeichnet: Meistens werde mit Essentialismus "falsche Universalisierung " gemeint.

Teresa de Lauretis hingegen bringt eine andere mit dem Lesbentum verbundene Diskussion auf, indem sie den Essentialismus mit dem sog. cultural feminism bzw. mit Separatismus und mit lesbischer Erfahrung überhaupt verbindet, und den Anti-Essentialismus als Angriff auf verschiedene Aspekte des radikalen (lesbischen) Feminismus interpretiert. In einer interessanten Diskussion über mehrere postmodeme (d.h. anti-essentialistische) feministische Texte ${ }^{12}$ behauptet sie z.B., $\mathrm{da} B$ diese Texte eigentlich alles auf eine Dichotomie zwischen Postmoderne und (radikalem) Feminismus reduzieren. Daraufhin schlägt sie ihre eigene Interpretation vor: Sie sieht in dem Versuch des Feminismus, sich selbst zu repräsentieren, zwei gleichzeitige Antriebe: einen erotischen, der u.a. Rebellion, Differenz, Subversion, agency behauptet und die Bilder von Machtlosigkeit, Opfersein, Passivität, Feminität ablehnt und einen ethischen, der sich mit »Sisterhood «, Koalition, gemeinsamer Verantwortlichkeit und gemeinsamen Tun befaßt (eventuell das Bild des Wirs, der Gemeinschaft). (266)

Neuerlich werden auch von der Seite der identity politics her Bedenken geäußert, da $\beta$ wir uns im Partikulären verlieren und dabei politisch verkommen. Eigentlich ist dies eine Ergänzung der anti-essentialistischen Diskussion: Während hier der Drang nach dem Partikulären kritisiert wird, plädieren die Anti-Anti-Essentialistinnen dort für Kategorien, für das politische Potential, das Verallgemeinerungen ermöglichen soll. Das heißt, als (meist akademische) Feministinnen, die auch ein politisches Programm haben, sind wir zu einem Balanceakt gezwungen: Wir wollen Differenzen anerkennen, wir wollen aber gleichzeitig politisch han- 
deln, und dafür brauchen wir eine Gemeinschaft, eine Koalition, die im Interesse von unseren politischen Zielen die Differenz weniger betonen soll.

Das Problem mit identity politics wird beschrieben als eine zu minutiöse Differenzierungsweise, durch die alles nur zur Vereinzelung führt. So fragt z.B. Christine Di Stefano: "Which groups do and do not qualify for specified categorical status, and on what grounds? At what point does theoretical analysis give way to ethnographic reporting? At what point do we all become endlessly particular individuals, each with a particular story to tell? ${ }^{13}(92)$ Lauter Ichs also. Und obwohl wir uns auch mit einer theoretischen Konzeptualisierung des Partikulären befassen, d.h. obwohl wir versuchen, diese Entwicklung theoretisch zu umschreiben, geht dabei etwas von der Koalitionspolitik verloren. Linda Gordon beschreibt, was sie die "politically constricting, even paralyzing effect to the multiplication of separate categories of oppression and victimization « nennt, wobei "political correctness means respecting the boundaries of the other categories and not crossing the lines-hardly a political atmosphere that promotes a democratic discourse or expands our visions of a good society. ${ }^{14}$ (106) Im extremsten Fall könnte eine in diesem Sinne verstandene identity politics bzw. difference bewirken, daß wir nicht mehr miteinander kommunizieren könnten: Das heißt auch, eine immer größer werdende Lücke wird entstehen zwischen Theorie und Praxis, zwischen der Universität und dem politischen Aktivismus.

Die Partikularisierung hängt zusammen mit dem Standpunkt/Ort/location, mit der Politik des Ortes: die bewußte Differenzierung, die stattfindet, wenn wir uns als einzelne Menschen betrachten. Aber wenn ein solcher Schritt gleichzeitig nicht nur die Möglichkeit der Zusammenarbeit infragestellt, sogar gefährdet, sondern auch die Verwischung anderer problematischer Fragen wie etwa die um Machtverhältnisse oder politische Tätigkeit (oder ganz einfach gesagt, die besondere Bedeutung des Genders als analytischer Kategorie für feministische Forschung) erlaubt, gibt das zu denken. Auch hier, wie bei den Beschuldigungen des Anti-Essentialismus, kommt die Befürchtung auf, daß wir verlieren, ohne etwas zu haben, womit wir das alles ersetzen können. Wie Susan Bordo in einem sehr guten Aufsatz über »Feminism, Postmodernism, and Gender Scepticism « gesagt hat, »If generalization is only permitted in the absence of multiple inflections or interpretive possibilities, then cultural generalizations of any sort...are ruled out. What remains is a universe composed entirely of counterexamples, in which the way men and women see the world is purely as particular individuals, shaped by the unique configurations that form that particularity. « ${ }^{15}$ (151)

Im Grunde hängt die identity politic mit der Überzeugung zusammen, daß Gender als einzige analytische Kategorie auch in den feministischen Diskussionen nicht genügt. Die anderen Kategorien wie Rasse, Klasse, sexuelle Orientierung etc. werden zunehmend ein Teil des Diskurses. Jedoch, wenigstens für die AntiAnti-Essentialistinnen wie de Lauretis und Frye, soll Gender seine analytische Bedeutung nicht verlieren. Und weil sie radikale lesbische Feministinnen sind, gibt es natürlich auch eine politische Ebene: Die Gefahr einer allzudetaillierten Partikularisierung bringt eine Individualisierung (das schöne amerikanische Ideal) mit sich, die politisch nicht mehr tragfähig ist. Der Ruf, etwas vorsichtiger mit den identity politics umzugehen, hat bestimmt auch mit der allgemeinen politi- 
schen Lage in den USA zu tun: Wir wählen immer in den USA, scheint mir, das kleinere Übel, aber gleichzeitig brauchen wir Koalitionen, damit wir das größere Übel nicht bekommen. ${ }^{16}$

Heißt das, daß wir in die Richtung der 1970er Jahre, also zur "Sisterhood" zurückkehren? Eher wollen wir die vielen politischen Fragen, die von identity politics ironischerwe ise manchmal übersehen wird, stellen. In diesem Zusammenhang ist auch das Theorie-Praxis-Problem angesiedelt. Die Theorie als solche, insbesondere die verallgemeinernden Aspekte der Theorie, die sehr oft von "women of color « angegriffen werden (siehe Barbara Christian, bell hooks, Gloria Anzaldúa u.a.) - ein Angriff, der bestimmt mit Fragen von inside und outside zusammenhängt - wird als Verengung angesehen, eine Art anti-identity politics und deren Konzept der Differenz. So zum Beispiel Gloria Anzaldúa, die über die TheoretikerInnen, die die sogenannte "queer theory« entwickeln, sagt, daß sie »theorize, that is, perceive, organize, classify and name specific chunks of reality by using approaches, styles and methodologies that are Anglo American or European. Their theories limit the ways we think about being queer. ${ }^{17}$ (251) Barbara Christian hat (in ihrem zum klassischen Modell gewordenen Aufsatz »The Race for Theory «) versucht, zwischen der rigiden, engen, einstimmigen Kategorie »der « Theorie und dem mehrstimmigen, komplexen, beweglichen, reichhaltigen Proze $B$ der theoretischen Konzeptualisierung zu unterscheiden. Die Grenzen überspringen, die Kategorien auflösen: Das machen zur Zeit in den USA vor allem feministische »women of color «, die ja immer zwischen den Stühlen von gender und race sitzen, oder Frauen aus dem Arbeiterstand, oder andere Randgruppen wie Lesben. Ihr Outsider-Status macht ihnen besonders klar, wie wichtig es ist, Grenzen, zwischen Theorie und Praxis, auch zwischen der Universität und der übrigen Welt, zu überwinden: bell hooks, zum Beispiel, plädiert immer wieder für eine Theoriebildung, die den Feminismus auf konkrete Weise unterstützt: »a theory that informs, shapes, and makes feminist practice possible.« (»Theory«, 5)

In der amerikanischen feministischen Diskussion heute geht es also um Identität, um die Frage, die uns seit mindestens dem 19. Jahrhundert beschäftigt, der Zeit der ersten Frauenbewegung: Wer sind wir als Frauen, als Feministinnen? Von welchem Standpunkt aus reden wir? Aber gerade dann, wenn »Identität « nicht als starre Abgrenzung verstanden wird, sondern als ProzeB, ist bei aller Differenz das Wechselseitig-aufeinander-bezogen-sein entscheidend. Nur dann kann es auch um gemeinsame Ziele gehen.

\section{Anmerkungen}

$1 \mathrm{Vgl}$. auch Lorraine Bethels Titel ihres 1979 erschienenen Aufsatzes "What Chou Mean We, White Girl?»

2 Vgl. den Aufsatz von Adrienne Rich, "Notes Toward a Politics of Location «.

3 Das scheint mir übrigens immer noch manchmal der Fall in Deutschland zu sein, denn bis jetzt hat der Ferminismus in den Hochschulen Deutschlands weniger FuB gefaßt als in den USA, die ja schon zu Beginn der 70er Jahre die Anfänge einer Institutionalisierung des Feminismus erlebt haben. 
4 Eine Theorie der Marginalität, der Subversion, der Opposition und des Anders-Sein-Auferlegens [othering] ... eine Theorie der sexuellen Grenzen ...

5 Orte der radikalen Offenheit ... Ohne solche Orte würden wir nicht überleben. Unser Leben beruht darauf, daß wir Alternativen (oft improvisierte) begrifflich formulieren können. Über diese Erfahrung ästhetisch und kritisch zu theoretisieren, bietet ein Programm für radikale kulturelle Praxis.

6 Es gibt mehrere Studien in Amerika, die sich mit verschiedenen Aspekten der sogenannten "feminist standpoint epistemologies« befassen: vgl. insbesondere Harding, Hartsock und Haraway. Für mich jedoch wird die Bezeichnung "Standpunkt " mit einer Betonung auf Partikularisierung in Zusammenhang gebracht. Was mich hier interessiert, ist eher die breitere Frage nach dem On und die daraus entstehenden besonderen Schwierigkeiten, wenn wir die Partikularisierung der »identity politics« mit der allgemeinen (eigentlich essentialistischen) Frage des Frauseins in Verbindung bringen.

$7 \mathrm{Ja}$, es kann sein, daß wir Menschen desselben Geschlechts lieben, aber wir sind nicht gleich.

8 Ich will diese Selbstbezeichnung, damit die Chicana und die Lesbe und die übrigen Menschen in mir nicht ausradiert, ausgelassen oder umgebracht werden. Das Selbstnennen gibt meine Anwesenheit bekannt, wie ich behaupte wer ich bin und auf welche Weise ich bekannt sein will. Das Selbstnennen ist eine Überlebensstrategie.

9 Ich mache mir Sorgen, daß der Standpunkt des weiblichen Anti-Essentialismus (wie manche Feministinnen ihn heute theoretisch fassen) ein nur für die privilegiertesten Frauen bestimmter Luxus ist. Ich mache mir Sorgen, daß dieser Standpunkt gleichbedeutend mit dem von manchen weißen männlichen Poststrukturalisten ist, die den Tod des Subjekts erklärt haben. Wenn Nancy $K$. Miller recht hat, wenn sie die Aussagen über den Tod des Subjekts in Frage stellt, indem sie insistiert, daß »nur diejenigen, die es haben, das Spiel wagen können, es nicht zu haben«, könnten wir nicht auch über antiessentialistische Feministinnen sagen, daß nur diejenigen, die weit mehr Möglichkeiten besitzen als die Mehrheit der Frauen, die heute in der Welt leben, das Spiel des "Es [d.h. Subjekt]-Seins « spielen können, ohne sich in den Glauben hinein zu theoretisieren, daß sie es nicht [d.h. daß sie kein Subjekt] sind?

Die Wortspiele in Modleskis Text sind eigentlich unübersetzbar.

10 Vicki Kirby und Elizabeth Grosz, zum Beispiel, in einem interessanten Sonderheft (1991) der Hypatia (der Zeitschrift in den USA für feministische Philosophie) über den Körper.

11 Negiert auch die Leistungen des Feminismus und die Wandlungsfähigkeit der Identität des Feminismus, die verschiedenen Bedeutungen, die diese Teilung innerhalb beweglicher historischer Kontexte markieren, zu negotiieren und infragezustellen.

12 Ihr Artikel setzt sich vor allem mit einem in der Zeitschrift Signs: Journal of Women in Culture and Society erschienenen Aufsatz von Linda Alcoff auseinander, nämlich „Cultural Feminism versus Post-Structuralism: The Identity Crisis in Feminist Theory « (Signs 13, 3 [Spring, 1988], S. 405-436). Auch kritisiert wird Chris Weedon (1987): Feminist Practice and Poststructuralist Theory. Oxford, New York.

13 Welche Gruppen werden für einen besonderen kategorischen Status ausgezeichnet, welche nicht? Und aus welchen Gründen? Wann weicht die theoretische Analyse dem ethnographischen Bericht? Wann werden wir alle unendlich partikuläre Individuen, jedes mit einer eigenen Geschichte, die es erzählen will?

14 Die politisch einengende, sogar lähmende Wirkung der Multiplizierung von getrennten Kategorien der Unterdrückung und der ungerechten Behandlung ... dabei heißt spolitical correctness eine Beachtung der Grenzen durch andere Kategorien und ein Nichtüberschreiten derselben kaum eine politische Atmosphäre, die einen demokratischen Diskurs fördert oder unsere Vorstellungen einer guten Gesellschaft erweitert.

15 Wenn Verallgemeinerung nur erlaubt wird, wenn mehrseitige Bedeutungen oder interpretative Möglichkeiten fehlen, dann werden kulturelle Verallgemeinerungen gänzlich ausgeschlossen. Was übrig bleibt, ist ein Universum, das nur aus Gegenbeispielen besteht, indem die Perspektiven von Männern und Frauen nur auf dem Standpunkt einzelner Individuen beruhen, die durch die einmaligen Konfigurationen geprägt werden, die jene Partikularität bilden.

$16 \mathrm{Vgl}$. Bernice Johnson Reagon: "You don't do no coalition building in a womb. (359)

17 Theoretisieren, d.h. sie erkennen, organisieren, klassifizieren und nennen vereinzelte Blöcke 
der Realität, indem sie Ansätze, Stile und Methoden benützen, die angloamerikanisch oder europäisch sind. Ihre Theorien wirken einengend auf die Denkweisen und Perspektiven des "Queer «-seins.

\section{Literatur}

Anzaldúa, Gloria (1991): To(o) Queer the Writer - Loca, escritora y chicana In: Warland, Betsy (Hrsg.): In Versions: Writings by Dykes, Queers \& Lesbians. Vancouver, S. 249-263.

Bethel, Lorraine (1979): What Chou MeanWe, White Girl? In: Conditions: Five 11, 2, S. 86-92.

Bordo, Susan (1990): Feminism, Postmodernism, and Gender-Scepticism. In: Nicholson, Linda J. (Hrsg.): Feminism/Postmodernism. New York/London, S. 133-156.

Carby, Hazel V. (1991): Feminism and the Politics of Difference, unv. MS.

Christian, Barbara (1989): The Race for Theory. In: Kauffman, Linda (Hrsg.): Gender and Theory. Dialogues on Feminist Criticism. Oxford, S. 225-237.

de Lauretis, Teresa (1990): Upping the Anti (sic) in Feminist Theory. In: Hirsch, Marianne und Evelyn Fox Keller (Hrsg.): Conflicts in Feminism. New York/London, S. 255-270.

Di Stefano, Christine (1991): Who the Heck Are We? Theoretical Turns Against Gender. In: Frontiers, XII, 2, S. 86-129.

Frye, Marilyn (1991): unv. MS., unbetitelte Arbeit über Ontologie, feministische Theorie und Essentialismus.

Fuss, Diana (1991): Inside/Out. In diess. (Hrsg.): Inside/Out. Lesbian Theories, Gay Theories. New York/London, S. 3-10.

Gordon, Linda (1991): On >Difference . In: Genders 10, S. 91-111.

Grosz, Elizabeth (1990): Conclusion: A Note on Essentialism and Difference. In: Gunew, Sneja (Hrsg.): Feminist Knowledge: Critique and Construct. New York/London, S. 332-344.

Grosz, Elizabeth (1991): Introduction. In: Hypatia 6, 3: Special Issue: Feminism and the Body, S. $1-2$.

Harding, Sandra (1986): The Science Question in Feminism. Ithaca/London.

Hartsock, Nancy (1983): Money, Sex and Power: Toward a Feminist Historical Materialism. Boston.

Haraway, Donna (1988): Situated Knowledges: The Science Question in Feminism and the Privilege of Partial Perspective. In: Feminist Studies 14, 3, S. 575-599.

hooks, bell (1990): Choosing the Margin as a Space of Radical Openness. In diess.: Yearning. Race, Gender and Cultural Politics. Boston, S. 145-154.

hooks, bell (1991): Theory as Liberatory Practice. In: Yale Journal of Law and Feminism 4, 1, S. $1-12$.

Kirby, Vicki (1991): Corporeal Habits: Addressing Essentialism Differently. In: Hypatia 6, 3, S. 4-24.

Miller, Nancy K. (1991); Getting Personal. Feminist Occasions and Other Autobiographical Acts. New York/London.

Modleski, Tania (1991): Feminism Without Women. Culture and Criticism in a »Postfeminist Age. New York/London.

Pratt, Minnie Bruce (1984): Identity: Skin Blood Heart. In: Bulkin, Elly und Minnie Bruce Pratt, Barbara Smith (Hrsg.): Yours in Struggle: Three Feminist Perspectives on Anti-Semitism and Racism. Ithaca, S. 11-63.

Reagon, Bernice Johnson (1983): Coalition Politics: Turning the Century. In: Smith, Barbara (Hrsg.): Home Girls. A Black Feminist Anthology. New York, S. 356-368.

Rich, Adrienne (1986): Notes toward a Politics of Location (1984). In diess.: Blood, Bread, and Poetry. Selected Prose 1979-1985. New York/London, S. 210-231. 